\title{
Ultrasonic Signal Reconstruction Using Compressed Sensing
}

\author{
Ren-Jean Liou \\ Department of Computer and Communications, National Pingtung University \\ 51 Mingshen E. Rd, Pingtung City, Taiwan, 900, ROC \\ renjean@mail.nptu.edu.tw
}

Keywords: SHM, interpolation, sparse representation, compressed sensing, $\ell_{1}$-norm

\begin{abstract}
Ultrasonic signal reconstruction for Structural Health Monitoring is a topic that has been discussed extensively. In this paper, we will apply the techniques of compressed sensing to reconstruct ultrasonic signals that are seriously damaged. To reconstruct the data, the application of conventional interpolation techniques is restricted under the criteria of Nyquist sampling theorem. The newly developed technique - compressed sensing breaks the limitations of Nyquist rate and provides effective results based upon sparse signal reconstruction. Sparse representation is constructed using Fourier transform basis. An $\ell_{1}$-norm optimization is then applied for reconstruction. Signals with temperature characteristics were synthetically created. We seriously corrupted these signals and tested the efficacy of our approach under two different scenarios. Firstly, the signal is randomly sampled at very low rates. Secondly, selected intervals were completely blank out. Simulation results show that the signals are effectively reconstructed. It outperforms conventional Spline interpolation in signal-to-noise ratio (SNR) with low variation, especially under very low data rates. This research demonstrates very promising results of using compressed sensing for ultrasonic signal reconstruction
\end{abstract}

\section{Introduction}

Ultrasonic inspection is an effective tool for flaw detection/evaluation, material characteristic measurement, and environmental monitoring. The typical principle is to transmit ultrasonic signal through the material and receive the transmitted wave at a distance for evaluation. Qualitative and non-continuous methods have long been used for the above tasks. Many applications do require continuous evaluation and monitoring for performance. Therefore, Structural Health Monitoring (SHM) has emerged and attracted significant attention in recent years for its wide potential of applications.

An SHM system is inevitably subjected to environmental and operational variations because of its in situ and in service nature of operation [1]. Low sampling rate and missing data due to sensor or system errors are common reasons that yield incomplete data during acquisition. It is possible to use digital signal processing techniques to reconstruct data. In addition to numerous conventional techniques, compressed sensing [2-4] becomes an effective approach recently for data reconstruction. Traditionally to achieve highly accurate signal analysis, a very high sampling rate is normally applied. Shannon-Nyquist sampling theorem are widely recognized and used. However, in many cases the signals are oversampled which may incur unnecessary hardware expenses. Compressed sensing imposes a new idea that the information rate of a continuous time signal is much lower than its bandwidth presented in the signal. Nyquist rate is hence sufficient but not necessary. Compressed sensing is an efficient approach for reconstruction of sparse signal from random samples. Numerous works have demonstrated its effectiveness in various applications, including ultrasonics [5-7].

This paper will illustrate the effectiveness of compressed sensing in the reconstruction of corrupted ultrasonic signals. Signal interpolation is indeed an reconstruction problem. We will first test data that were randomly selected from the original signal. Then we will test data with some intervals completely removed. These data are all with very low data rates. Simulation results show that, in compared to conventional Spline method, compressed sensing with sparse reconstruction provides extraordinary performance in both scenarios. 
This paper is organized as follows: we first illustrate how ultrasonic signal was generated, then describe the method of compressed sensing; simulation results are then given to demonstrate the effectiveness in signal reconstruction; finally, conclusion is provided.

\section{Ultrasonic Signal Generation}

In ultrasonic signal analysis, the only common environmental effect that has been successfully investigated is temperature. Michaels and Michaels [8] employed the local temporal coherence to discriminate damage from temperature changes using diffuse ultrasonic guided waves in SHM. Ultrasonic signals with various temperatures changes in certain regular patterns. Michaels [1][8] have discovered this regularity. This methodology is based upon the prediction and observation that the first order effect of a temperature changed on a diffuse ultrasonic wave is a time dilation or compression.

This paper is aimed on signal reconstruction of ultrasonic lamb wave in the temperature domain. Signals are synthetically generated based upon the characteristics of temperature change. We first assume aluminal material is used. Its thickness is then defined. The corresponding longitudinal and transversal speeds with various temperatures can hence be calculated. The package of AGU-Vallen Wavelet [9] was then used to generate the dispersion curve, which can be converted to $\omega-k$ curves for the desired Synchronous and Asynchronous modes. Ultrasonic signals can thus be simulated based upon these setups.

\section{Compressed Sampling for Signal Reconstruction}

Conventional approaches for data sampling are limited by Shannon's theorem or the Nyquist criteria. The sampling rate must be at least twice the bandwidth of the signal in order to avoid the phenomena of aliasing. This theorem dominates nearly all signal acquisition implementations including audio, imaging and so forth. Nyquist rate is indeed so high that many samples are generated, making compression a necessity prior to storage or transmission. In addition, high-speed $\mathrm{A} / \mathrm{D}$ and $\mathrm{D} / \mathrm{A}$ converters are needed, which makes the sampling process very expensive.

Compressed sensing was proposed in recent years to capture and represent compressible signals at a rate much below the Nyquist rate. It employs nonadaptive linear projections that preserve the structure of the signal. The signal is then reconstructed from these projections using an optimization process [10-13]. Acquisition of data samples can be replaced only by a few measurements. The effectiveness of compressed sensing is determined by the properties of sparsity and incoherence.

Many natural signals can have concise representations when they are expressed in a proper basis $\psi$. This is called sparsity. Sparsity expresses the idea that the information rate of a continuous time signal may be much smaller than its bandwidth. The basis includes Fourier, wavelet, or various kinds of transform matrices. There are also a number of approaches to find the basis iteratively [14-15]. To have good representation of data space, objects that have a sparse representation in $\psi$ must spread out in the domain in which they are acquired. This is the feature of incoherence.

Consider a finite-length, discrete-time signal $x[n]$, with $n=1,2 \ldots N$. Any signal of $x[n]$ can be represented in terms of a basis $\psi_{i}$, which is a column vector. For simplicity, we should select the basis that is orthonormal. Let $\mathbf{X}$ represents the signal in vector form, it can then be expressed as

$$
\mathbf{X}=\sum_{i=1}^{n} \alpha_{i} \psi_{i} \quad \text { or } \quad \mathbf{X}=\mathbf{\Psi} \boldsymbol{\alpha}
$$

where $\alpha$ is the $N \times 1$ column vector of weighting coefficients $\alpha_{i}=\psi_{i}^{T} \mathbf{X} .{ }^{T}$ denotes transposition. Hece $\mathbf{X}$ and $\alpha$ are equivalent representations of the signal. $\mathbf{X}$ is in the time or space domain and $\alpha$ is in the $\Psi$ domain. If only $K$ of the coefficients $\alpha_{i}$ are nonzero or relatively large, we say that signal $\mathbf{X}$ is $K$-sparse. When $K<<N$, the signal is compressible. In many transform coding algorithms, the $K$ largest coefficients are kept and the rest $N-K$ coefficients are discarded. The sampling-then-compressing framework is very inefficient. Compressed sensing can address this 
problem by directly acquiring a compressed signal of $K$ measurements instead of acquiring all $N$ samples.

The essence of compressed sensing is to acquire condensed data through dimensionality reduction. We can apply measurement matrix $\Phi$ and compute $M$ inner products between $\phi_{i}$ and $\mathbf{X}$. This will yield an $M \times 1$ vector $\mathbf{Y}$ which is the measurement. Hence we have

$$
\mathbf{Y}=\boldsymbol{\Phi X}=\boldsymbol{\Phi} \boldsymbol{\Psi}_{\alpha}
$$

Now $\Phi \Psi$ is an $M \times N$ matrix. In order to design a stable measurement matrix, it can be shown that for a small constant $c$,

$$
M \geq c K \log (N / K)
$$

A Gaussian matrix is convinient to define the measurement matrix. To proceed, we need to design a signal reconstruction algorithm based upon equation (2). It is indeed similar to an inverse problem. We shall apply $\ell_{\mathrm{p}}$-norm minimization to find the solution.

The $\ell_{\mathrm{p}}$-norm is defined as

$$
\|s\|_{p}=\left(\sum_{i=1}^{N}\left|s_{i}\right|^{p}\right)^{\frac{1}{p}}
$$

The solution of reconstruction can be found as

$$
\min _{\alpha \in R^{N}}\|\hat{\alpha}\|_{p} \text { subject to } \mathbf{Y}=\mathbf{\Phi X}
$$

It was shown in many compressed sensing literatures that minimum $\ell_{0}$-norm is numerical unstable and NP-complete. Minimum $\ell_{2}$-norm can never find a $K$-sparse solution, returning a nonsparse $\hat{\alpha}$ with many nonzero elements. As a consequence, minimum $\ell_{1}$-norm is a convex optimization problem. It conveniently reduces the problem to a linear programing and provides efficient solution algorithm. The reconstructed signal $\hat{x}$ is then given by $\hat{x}=\Psi \hat{\alpha}$.

The key to success of using compressed sensing for ultrasonic signal reconstruction is the choice of basis $\Psi$. The most commonly used basis is the Fourier transform matrix. Since most signals are band-limited, converting signals into the frequency domain will have clear view of the signal characteristics. The concentration of signal in the frequency domain makes the signal sparse. Compressed sensing algorithm can hence be applied.

\section{Simulation Results}

To verify the efficacy of compressed sensing in ultrasonic signal reconstruction tests were conducted. Temperature signals were synthetically generated for implementation. Processing is performed in time domain. Temperature at $0^{\circ} \mathrm{C}$ is used as references. The sampling frequency is $20 \mathrm{MHz}$. The signal center frequency is $100 \mathrm{KHz}$. Hence the minimal sampling rate should be at least $200 \mathrm{KHz}$, which is the Nyquist rate. The data length is $1 \mathrm{~ms}$, which is equivalent to 20,000 samples in time. Methods of compressed sensing and spline interpolation were applied to compare the reconstruction performance. Simulations are implemented with two kinds of scenarios. The first scenario is to randomly select data points at limited percentage. The second scenario is to periodically remove data points at every fixed length of interval. Reconstructions using methods of compressed sensing and spline interpolation are performed on these two incomplete data sets for comparison. Fourier matrices are used as basis in compressed sensing. Spectral Projected Gradient for $\ell_{1}$-norm minimization [16] is applied for optimal reconstruction.

In the first scenario, only 600 random samples were selected during the $1 \mathrm{~ms}$ period. The data rate is only 3\%. Due to random selection, the sampling rate is considerablely lower than the Nyquist rate at some points. Figs. 1 and 2 shows the results of applying compressed sensing with sparse reconstruction and spline interpolation, respectively. For ease of observation, only time segments between 200 and $300 \mu$ s are shown. The original signal is shown in blue. Green dots represent the randomly selected data points. We can see that the result of compressed sensing closely follows the signal center frequency. Major errors usually occur at the peak amplitude. In general the result of spline interpolation is smoother and more accurate as long as sampling rate is high enough. Hence the 
blue and red curves in Fig. 2 have higher overlap than in Fig. 1. However, this method fails completely when sampling rate is too low. This can be seen at around $240 \mu$ s. The reconstructed signal is shown in red. The SNRs of reconstructed signals using compressed sensing and spline are $13.9 \mathrm{~dB}$ and $6.44 \mathrm{~dB}$, respectively.

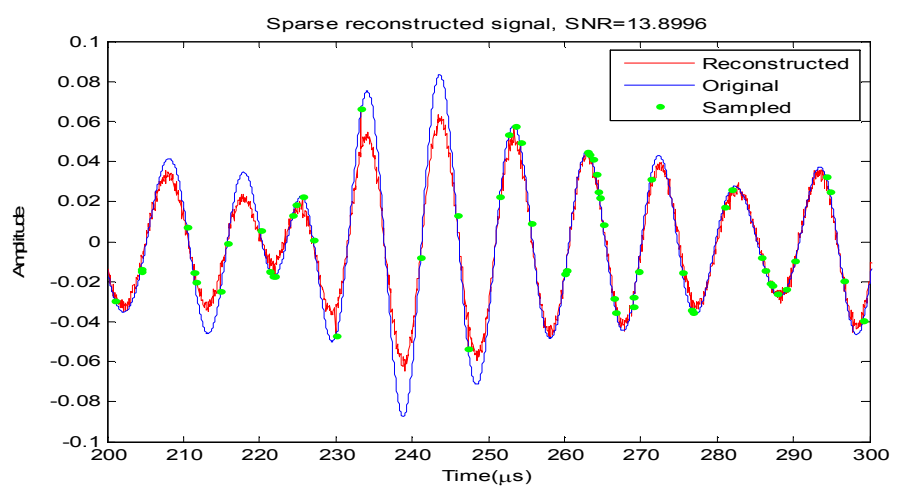

Fig. 1 Reconstruction of 600 randomly selected samples using compressed sensing. (Enlarged)

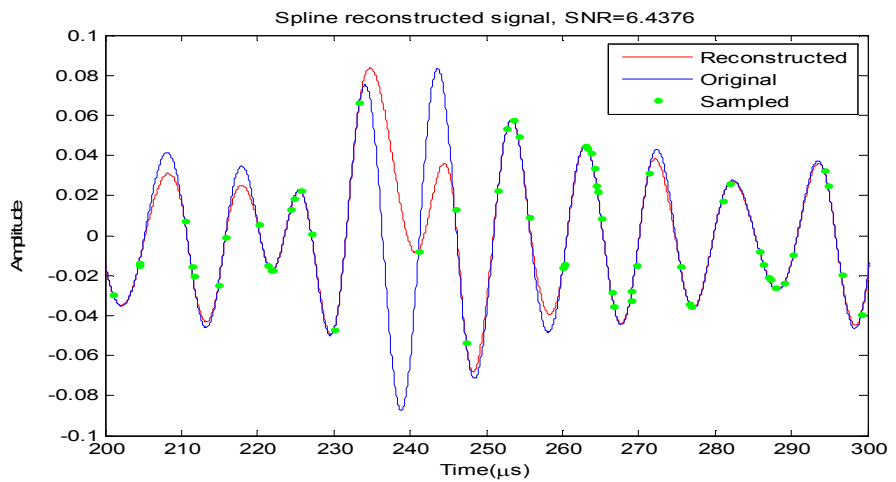

Fig. 2 Reconstruction using Spline interpolation with same scenario as in Fig. 1.

In scenario 2, original signal is equally divided into 20 sections. Each section has time duration of $500 \mu \mathrm{s}$ or equivilantly1,000 points in length. This scenario simulates the situation of sensor transmission errors. When system malfunction occurs during signal acquisition, data taking may stop for periods of time. Figs. 3 and 4 shows the results of applying compressed sensing with sparse reconstruction and spline interpolation, respectively. Again, the original signal is shown in blue. Green dots represent the selected data points for reconstruction. The reconstructed signal is shown in red. The SNR of reconstructed signals using compressed sensing reaches as high as $22.06 \mathrm{~dB}$. This is extraordianry and even much better than the case in Fig. 1. The major reason is that more data points are presented in this simulation while the previous one has much lower data rates. Spline method only has SNR of $-1.29 \mathrm{~dB}$. It is even worse than that in Fig. 2. It completely failed within the periods where data is missing.

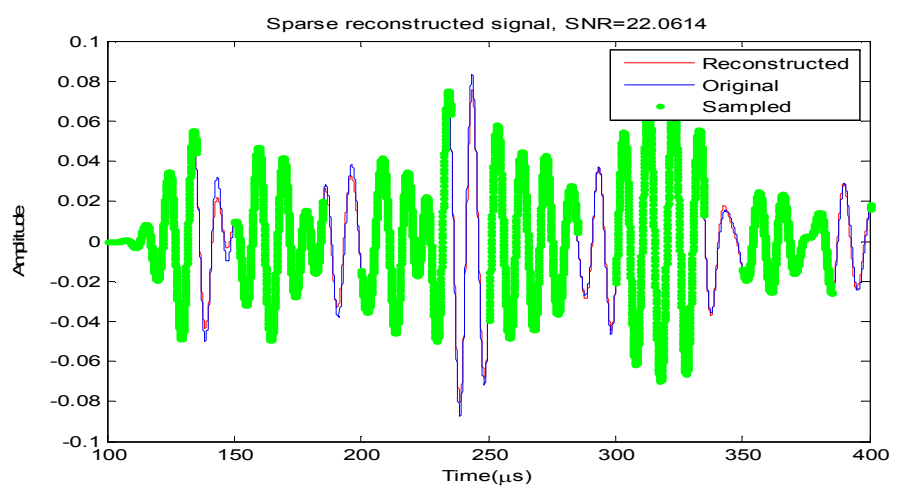

Fig. 3 Reconstruction of periodically selected samples using compressed sensing. 


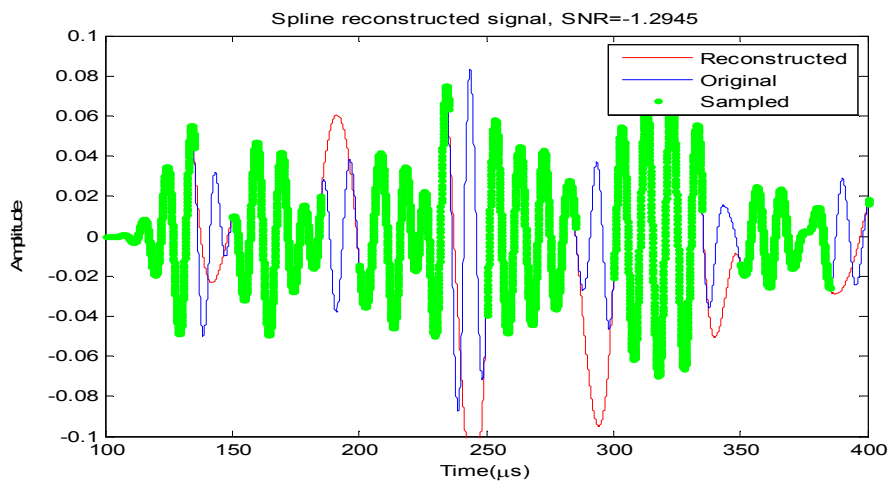

Fig. 4 Reconstruction using Spline interpolation with same scenario as in Fig. 4.

To further verify the consistency of performance, we repeatedly test the data sets with different number of selected samples. To reduce the computational complexity, continuous 4800 of the original data samples were extracted. This is equivalent to $240 \mu$ s in length. Simulations using compressed sensing and spline interpolation were repeated 50 times with randomly selected data from these 4800 samples. Differenct random samples are selected in each time. We calculated the mean and variance of SNR and compared their performance.

Table 1 shows the experimental results. Column 1 is the total number of samples $M$ selected for reconstruction. When $M=100$, it means that only 100 points were randomly selected during this $240 \mu \mathrm{s}$ period, or from the 4800 samples. The average sampling period is hence $2.4 \mu \mathrm{s}$, which is equivalent to $417 \mathrm{KHz}$. Since the samples are randomly selected, many times the sampling frequency is well below $417 \mathrm{KHz}$ and even below the Nyquist rate.

In the table, we can see that for lower $M$, the average performance of compressed sensing is better than spline interpolation. This is more evident when $M$ is small. The mean SNR using compressed sensing reaches $20 \mathrm{~dB}$ as $M$ approaches 200 . The mean SNR of spline interpolation is higher only when $M$ is big enough, i.e., when the required sampling rate was achieved. Variance defines the consistency of performance among the 50 trials. It is noteworthy that the performance of compressed sensing is more stable as it always gives lower variance. It shows that the performance of spline is rather data-dependent as it has high variance due to randomly selecteds amples. The last row shows that even when $M$ reaches as high as 600 , method of spline still has high variance of $43 \mathrm{~dB}$. This means that the SNR can go down to less than 10dB. This is among the worst cases.

Table 1 SNR and variance comparison of compressed sensing (CS) and spline interpolation.

\begin{tabular}{|c|c|c|c|c|}
\hline$M$ & $\begin{array}{c}\text { CS mean } \\
\text { SNR }\end{array}$ & $\begin{array}{c}\text { CS SNR } \\
\text { variance }\end{array}$ & $\begin{array}{c}\text { spline } \\
\text { mean SNR }\end{array}$ & $\begin{array}{c}\text { spline SNR } \\
\text { variance }\end{array}$ \\
\hline 100 & 6.45 & 2.19 & -0.02 & 25.56 \\
\hline 150 & 14.55 & 13.08 & 9.27 & 35.56 \\
\hline 200 & 20.16 & 6.35 & 17.14 & 27.82 \\
\hline 300 & 25.11 & 1.58 & 28.54 & 46.92 \\
\hline 600 & 32.24 & 3.5 & 51.78 & 43.12 \\
\hline
\end{tabular}

\section{Conclusions}

Ultrasonic signal has wide applications in material charactrization and monitoring. However, signal acquisition is often destructed by environmental condition. A good signal reconstruction is important for consistent performance. This paper applied compressed sensing for reliable data reconstruction. It breaks the limitation of Nyquist creteria and acquire data at very low sampling rate. Remarkable perfomance is achieved with data rate below $3 \%$ at sampling rate of $20 \mathrm{MHz}$. We tested the algorithm on two scenarios: randomly selected samples and periodically selecting intervals of data. In random sampling, compressed sensing presents higher errors around wave peaks. In periodic sampling, compressed sensing properly replenishes data in the missing intervals. The method was compared to conventional spline interpolation with various numbers of data points. We can observe 
that compressed sensing gives higher SNR and lower variance especially at low sampling rate. The variance defines the consistency of performance. Our results justify that the proposed method has great advantages over some conventional methods especially when sampling rate is low.

\section{Acknowledgements}

The author would like to thank Dr. Jeniffer Michaels at the School of Electrical and Computer Engineering, Georgia Institute of Technology, USA for providing ultrasonic data in the simulations of this work.

\section{References}

[1] Y. Lu and J. E. Michaels, Feature extraction and sensor fusion for ultrasonic structural health monitoring under changing environmental conditions, IEEE Sensors Journal, 9-11 (2009) 1462-1471.

[2] D. Donoho, Compressed sensing, IEEE Trans. Inform. Theory, 52-4 (2006) 1289-1306.

[3] Richard G. Baraniuk, Compressive Sensing, IEEE Signal Processing Magazine, 4 (2007) 118-121.

[4] Emmanuel J. Candès and Michael B. Wakin, An Introduction To Compressive Sampling, IEEE Signal Processing Magazine, 2 (2008) 21-30.

[5] Guangming Shi, Chongyu Chen, Jie Lin, Xuemei Xie, and Xuyang Chen, Narrowband Ultrasonic Detection With High Range Resolution: Separating Echoes via Compressed Sensing and Singular Value Decomposition, IEEE Transactions on Ultrasonics, Ferroelectrics, and Frequency Control, 59-10 (2012) 2237-2253.

[6] Zhang Hua, Chen Xiao-Hong, and Wu Xin-Min, Seismic data reconstruction based on CS and Fourier theory, Applied Geophysics, 10-2 (2013) 170-180.

[7] Y.-J. Zhao, X.-Y. Zhuang, H.-J. Wang, and Z.-J. Dai, Ultrasonic signal compressive detection using improved random equivalent sampling, IET Science, Measurement and Technology, 6-4 (2012) 261-266.

[8] Y. Lu and J. E. Michaels, A Methodology for Structural Health Monitoring with Diffuse Ultrasonic Waves in the Presence of Temperature Variations, Ultrasonics, 43 (2005) 717-731.

[9] AGU-Vallen Wavelet, http://www.vallen.de/freeware 2012.

[10] E. Candès, J. Romberg, and T. Tao, Robust uncertainty principles: Exact signal reconstruction from highly incomplete frequency information, IEEE Trans. Inform. Theory, 52-2 (2006) 489-509.

[11] D. Donoho, Compressed sensing, IEEE Trans. Inform. Theory, 52-4 (2006) 1289-1306.

[12] Richard G. Baraniuk, Compressive Sensing, IEEE Signal Processing Magazine, 4 (2007) 118-121.

[13] Emmanuel J. Candès and Michael B. Wakin, An Introduction To Compressive Sampling, IEEE Signal Processing Magazine, 2 (2008) 21-30.

[14] S.S. Chen, D.L. Donoho, and M.A. Saunders, Atomic decomposition by basis pursuit, SIAM J. Sci. Comput., 20-1 (1998) 33-61.

[15] J. Tropp and A.C. Gilbert, Signal recovery from partial information via orthogonal matching pursuit, IEEE Trans. Inform. Theory, 53-12 (2007) 4655-4666.

[16] E. van den Berg and M. P. Friedlander, Probing the Pareto frontier for basis pursuit solutions, UBC Computer Science Technical Report TR-2008-01, 2008. 